\title{
Educación centrada en el crecimiento de la relación interpersonal
}

Education Centered on the Growth of the Interpersonal Relationship

JosÉ VÍCTOR ORÓN SEMPER

Universidad de Navarra

joron@unav.es
RECIBIDO: 22 DE JUNIO DE 2017

VERSIÓN DEFINITIVA: 28 DE SEPTIEMBRE DE 2017

DOI: $10.15581 / 013.20 .241-262$
Resumen: La forma de entender la educación realizó un giro de una educación centrada en el profesor a una educación centrada en el alumno. Si en el modelo centrado en el profesor el acto educativo descansa sobre todo en lo que el profesor realiza, en el modelo centrado en el alumno el acto educativo descansa sobre todo en lo que el alumno hace. No obstante, proponemos que este cambio es insuficiente y que hace falta más bien entender el acto educativo centrado en todo el juego de relaciones interpersonales que permite un centro escolar.

Palabras clave: Educación centrada en el alumno, modelos educativos, relaciones interpersonales.
Abstract: The way of understanding education shifted from a teacher-centered model to a student-centered one. While in the teacher-centered model the educational act rests primarily on what the teacher does, in the latter the educational act rests above all on what the student does. However, we propose that this change is insufficient and that it is necessary to understand the educational act as centered on all the possibilities of interpersonal relationships (relationship-centered model) that a school allows.

Keywords: Student-Centered Education, Educational Models, Interpersonal Relationships. 


\section{INTRODUCCIÓN}

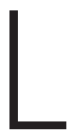
a forma de entender la educación ha realizado un giro de una educación centrada en el profesor a una educación centrada en el alumno. Con ese giro se ha realizado un cambio, al menos a nivel teórico. Si en el modelo de la educación centrada en el profesor, el acto educativo descansa sobre todo en lo que el profesor realiza, y lo más predominante era la exposición oral, en el modelo centrado en el alumno, el acto educativo descansa sobre todo en lo que el alumno hace.

Un punto de inflexión en este proceso se debió en gran medida gracias a Dewey ${ }^{1}$, quien señaló a la experiencia vivida por el alumno como el punto de arranque del acto educativo y no lo que el profesor hace. Otros autores han seguido esta propuesta y desde luego el principio de experiencia en ningún momento ha sido discutido. Por ejemplo, en la propuesta de Kolb² vemos una secuencia ordenada del aprendizaje que tiene como punto de partida la experiencia. Incluso en propuestas que se suelen calificar de excesivamente cognitivistas el principio de experiencia está claramente identificado3.

También desde la psicoterapia se ha alentado este cambio centrado en el alumno, en este caso centrado en el cliente, en su situación personal, y no tanto en los datos con que éste puede ser caracterizado ${ }^{4}$.

Este principio de experiencia no ha ignorado la importancia de las relaciones sociales en el proceso de crecimiento. En el mismo texto citado de Piaget se ve que el desarrollo cognitivo del niño sólo se produce en la medida que el fruto de su actividad sirve para la cooperación social. Otros autores han pasado a la historia como un referente de la importancia en las relaciones sociales como Vygostky o Paulo Freire.

Este principio de experiencia, si se exagera puede llevar a un modelo educativo que presenta problemas, pues parecería que el mero hecho de vivir ex-

${ }^{1}$ J. DEWEY, Experience and education, Collier Books, New York, 1938; J. DEWEY, "Having an experience", en Art as Experience, Perigee Books, New York, 1934, 371.

2 D. A. KOLB, Experiential Learning. Experience as the Source of Learning Development, Pearson Education, New Jersey, 2015.

3 J. PIAGET, "The rules of the game", en The Moral Fudgement of the Child, The Free Press, Glecone, 1965, 1-104.

${ }^{4}$ C. R. ROGERS, El proceso de convertirse en persona, mi técnica terapéutica, Paidós Ibérica, Barcelona, 2000.

5 L. S. VYGOTSKY, Mind in Society: The Development of Higer Psychological Processes, Harvard University Press, Cambridge (MA), 1978. 
periencias de por sí, podría ser garantía de que se está educando cuando no es así. Una exageración del principio de experiencia lleva a ignorar que la educación sólo se entiende en la inter-relación humana ${ }^{6}$. El principio de experiencia señala que algo tiene que vivir el alumno para que se dé su progreso escolar. Ciertamente la educación puede entenderse perfectamente como un "ayudar a crecer". Pero un excesivo centramiento en lo que tiene que ocurrir en el alumno puede llevar, y sospechamos que así ocurre, a pensar que nada tiene que pasar en el profesor o en la relación entre ambos, pues el que ha de cambiar es el alumno. En tal caso llegamos a un modelo de profesor que se comporta a modo de entrenador. Esto explicaría también el hecho de que observamos una creciente preocupación por la auto-regulación del alumno.

Para profundizar en el modelo educativo del entrenador o instructor fijémonos por ejemplo en un entrenador de caballos. Al principio y al final de la sesión de entrenamiento, el entrenador no ha cambiado personalmente, simplemente se ha cansado. En el entrenamiento está claro el punto de llegada al que ha de llegar el caballo con su entrenamiento. Normalmente éste está medido de forma comportamental porque el caballo ha adquirido una serie de habilidades. El entrenador de caballos dirige los acontecimientos y sirve de soporte para que el caballo realice su tránsito. Del caballo se espera obediencia, desarrollo de habilidades, cierta cercanía con el entrenador, etcétera. Con este modelo llegamos al caballo entrenado. Obviamente nadie acepta que lo que se tiene que hacer con los alumnos sea domarlos como a los caballos. Pero, de hecho, es un modo de pensar que podría reconocerse cuando lo que se busca es que el niño y luego joven se ajuste a la sociedad y a las demandas de trabajo que la sociedad tiene o cuando el punto de llegada está definido por unas competencias concretas y el profesor a modo de entrenador, apoya el desarrollo del alumno, pero él no tiene por qué cambiar. En tal caso surge la persona 'entrenada'. Pero como muy bien criticó Peters, lo que se busca es la persona 'educada', no la persona 'entrenada's.

Podríamos preguntarnos si esta mentalidad del entrenamiento se da o no se da como un derivado del principio de experiencia. Hay que señalar con claridad que los que han propuesto el principio de experiencia también han se-

${ }^{6}$ F. ALTAREJOS, C. NAVAL, Filosofía de la educación, Eunsa, Pamplona, 2000, 38.

${ }^{7}$ L. POLO, Ayudar a crecer: cuestiones de filosofía de la educación, Eunsa, Pamplona, 2006.

${ }^{8}$ R. S. PETERS, Ethics and Education, Routledge, New York, 1966, 34; cfr. también R. S. PETERS, "What is an educational process", en R. S. PETERS (Ed.), The Concept of Education, Routledge \& Kegan Paul, London, 1967, 1-23. 
ñalado la importancia de la educación social, pues de lo contrario no se tendría buenos ciudadanos?. Por tanto, ciertamente en la intención de Dewey está la preocupación por no caer en un principio exacerbado de experiencia. Pero lo que cabría preguntarse es si, al margen de su intención, su propuesta permite un desarrollo que conduzca a ello. Supera el alcance del artículo hacer tal estudio.

Hoy en día ese 'algo' que tiene que pasar en el alumno, se formula en clave de competencia. Por ejemplo, en España, la legislación de todo el sistema evaluativo y de programación escolar está en clave de competencias ${ }^{10}$. La educación busca que los alumnos sean competentes que es mucho más que hábiles. Si estamos en la mentalidad de que el alumno debe de prepararse para ser, por ejemplo, un buen herrero, surge la mentalidad de educar para tener habilidades. Tiene que ser hábil para trabajar el hierro. Las demandas de la revolución industrial mostraron la insuficiencia de la habilidad, pues hacía falta una versatilidad y creatividad que parece que la educación en la mera habilidad no daba tanto de sí. Así nació el término competencia ${ }^{11}$.

Un documento clave a nivel educativo es el Informe UNESCO: La educación encierra un tesoro ${ }^{12}$. En ese documento se recoge la palabra competencia aplicada a la educación, pero llama la atención que lo hace con cierto recelo, pues educar es más que ser competente. Allí podemos leer que entre "las tensiones que ban de superarse"13 se menciona la existente entre competencia e igualdad de oportunidades y se habla del riesgo de la presión de la competencia, pues "bace olvidar a muchos directivos la misión de dar a cada ser bumano los medios de aprovechar todas sus oportunidades" ${ }^{14} \mathrm{y}$ la solución es "retomar y actualizar el concepto de educación durante toda la vida" ${ }^{\prime 5}$.

El Informe UNESCO dice que la educación debe tener cuatro pilares sobre los que construirse y la competencia sólo se menciona en uno de los cua-

\footnotetext{
J. DEWEY, Experience and education, op. cit.

10 Gobierno de España, Ministerio de Educación Cultura y Deporte, "Orden ECD/65/2015, de 21 de enero, por la que se describen las relaciones entre las competencias, los contenidos y los criterios de evaluación de la educación primaria, la educación secundaria obligatoria y el bachillerato", Boletín Oficial del Estado, 2015, BOE-A-2015-738, 6986-7003.

11 J. DELORS et al., La educación encierra un tesoro. Informe UNESCO de la Comisión Internacional sobre la Educación para el Siglo XXI, Santillana-UNESCO, Madrid, 1996, 99-103.

12 Ibidem.

13 Ibidem, 16-18.

14 Ibidem, 17.

15 Ibidem, 17.
} 
tro pilares, "el aprender a hacer" y lo hace con una función instrumental a los otros tres ${ }^{16}$. Todavía más, la postura de la comisión es la de "insistir especialmente en uno de los cuatro pilares presentados e ilustrados como las bases de la educación. Se trata de aprender a vivir juntos ${ }^{\prime 17}$ esto es: conocer mejor a los demás para con un espíritu nuevo impulsar proyectos comunes teniendo presente que las relaciones de interdependencia son cada vez mayores. Por lo que puede decirse que no hay suma, sino jerarquía entre ellos. No puede aislarse un pilar de los otros pues la educación debe concebirse como un todo ${ }^{18}$.

Además, entre los diversos aprendizajes propuesto por la UNESCO (aprender a conocer y a aprender, aprender a hacer, aprender a vivir juntos y aprender a ser) sólo en uno de ellos (en aprender a hacer) aparece la palabra competencia. En cambio, observamos que muchos sistemas educativos hoy en día han asumido la educación en competencias como el principio rector. Por lo tanto, un sistema educativo basado en las competencias, no estaría respetando la propuesta de la UNESCO que propone como centro o base el aprender a vivir juntos insistiendo en la comprensión de las situaciones personales, la interdependencia y los valores ${ }^{19}$. Desde luego no se le puede achacar a la competencia que no cumpla el principio de experiencia, ni que se haya descentrado del alumno. La competencia es la competencia del alumno y es bien experiencial por las dimensiones prácticas que tiene el proceso de aprendizaje. Pensamos, en cambio, que un modelo educativo en clave competencial, es decir cuando se toma la competencia como el eje rector, presenta muchas limitaciones. La mayor de ella es que la competencia se evalúa fundamentalmente por la eficiencia de ella para resolver situaciones y por tanto el principio de evaluación está en algo exterior a la persona y no en algo interior a ella. Una persona es competente no por lo que el desarrollo de esa competencia ha supuesto para ella, sino por la repercusión que tiene la competencia fuera de la persona.

Pensamos que el modelo competencial ha sido de hecho una evolución de los principios de una educación centrada en el alumno y del principio de experiencia. En el presente artículo lo que se pretende mostrar son algunas li-

\footnotetext{
Ibidem, 99-103.

Ibidem, 21-22.

Ibidem, 109.

Ibidem, 103-106. Hay que tener presente que el término valores, en concreto valores morales, es entendido como en referencia a lo espiritual que incluiría lo religioso en el caso de que la persona lo fuera.
} 
mitaciones del modelo competencial, pero sobre todo, se pretende mostrar que hay otro modelo que puede dar mejor cauce a los deseos que motivaron la propuesta de centrarse en el alumno y en la experiencia. Este otro modelo podríamos llamarlo centrado en la relación interpersonal educador-alumno, siendo así una alternativa a los modelos centrado en el profesor o centrado en el alumno.

Para nuestra propuesta nos basaremos en el trabajo de diversos filósofos de la educación, sobre todo en Francisco Altarejos, que pensamos se encuentra en plena sintonía con el ensayo de filosofía de la educación de Leonardo Polo $^{20}$. Para dar robustez a la propuesta de Altarejos acudiremos a otros filósofos que han hecho sus aportaciones en la educación: Víctor García Hoz, Richard Stanley Peters, Alfred North Whitehead, Lawrence Kohlberg y Octavi Fullat.

Así pues el artículo continúa señalando elementos de los autores indicados que sirven para la propuesta de una educación centrada en la relación y luego haremos una formulación inicial de nuestra propuesta.

\section{Elementos FILOSÓfICOS QUE SIRVEN DE BASE PARA LA EDUCACIÓN CENTRADA EN LA RELACIÓN}

La propuesta educativa de Leonardo Polo deriva de su visión antropológica, sobre todo del carácter de sistema del ser humano, la apertura irrestricta y su ser persona ${ }^{21}$ :

a) El carácter de sistema obliga a rechazar cualquier tipo de parcelación independiente en la educación y a acoger una visión holística. Holístico no significa simplemente que se trate todo (eso sería comprensivo) sino que se trata todo unificada e interrelacionadamente sin posibilidad de independencia. Eso obliga no sólo a la unificación sistémica de lo que ocurre en el centro escolar, sino también en unificar la educación familiar, la escolar y la específica.

b) El crecimiento irrestricto del ser humano hace que la persona esté radicalmente abierta al crecimiento y por ello nunca llega a ser todo lo que pue-

${ }^{20}$ L. POLO, op. cit.

21 Un desarrollo de los mismos no corresponde ahora, pero las referencias podemos encontrarlas en L. POLO, Obras Completas XV. Antropología trascendental, Eunsa, Pamplona, 2016; Obras Completas X. Quién es el hombre. Presente y futuro del hombre, Eunsa, Pamplona, 2016; Ética. Hacia una versión moderna de temas clasicos, Aedos-Unión Editorial, Madrid, 1997. 
de ser. La educación no puede tener otro fin distinto de éste, el crecimiento se convierte en el verdadero tema de cada ser humano. Por ser irrestricto la educación no viene a satisfacer una necesidad, sino a hacer posible esta llamada interior, por ello el ser humano necesita formación. Mientras que un animal simplemente gana en eficiencia, el ser humano se va formando, pues el crecimiento es formativo, afecta a lo que es. Por ello, la educación no es algo circunscrito a un periodo vital, sino que se extiende a lo largo de toda la vida ${ }^{22}$. Si la educación no busca algo exterior a la persona, sino su propio crecimiento, quiere decir que la educación se entiende como acto, no como producción. Siguiendo la división aristotélica el acto tiene sentido en sí mismo, pues alcanza su fin en el mismo acto, mientras que la producción tiene sentido por lo que ocurre al final ${ }^{23}$.

c) El carácter de persona remite a la singularidad de nuestra existencia debida a un acto particular. Detrás de esto hay una doble afirmación: todo ser humano es hijo y todo ser humano es co-ser. Somos seres co-existentes, se nace siendo sociedad. Sólo en el ser humano hay conciencia de relación filial con el origen. Por eso la educación no está ligada a una deficiencia de conocimiento, sino al hecho de ser hijo, lo cual se mantiene a lo largo de la vida. Esto hace que la educación pueda tomarse como paradigma de las relaciones interpersonales. Nos relacionamos para crecer. Todas nuestras relaciones deberían de ser educativas.

Para el propósito del artículo nos interesa destacar tres aspectos más: el sentido humano de la técnica, la familia y la educación de la afectividad, la imaginación y la motivación.

La postura de Polo sobre la técnica es importante porque puede ayudar a entender el lugar de la competencia en la educación. Polo tiene una alta consideración de la técnica pues el ser humano es un homo faber, por lo tanto, sin la técnica no hubiera habido evolución. La técnica podríamos considerarla en relación al radical moderno. Polo habla de tres radicales ${ }^{24}$, como de tres elementos para entender al ser humano y su actuar ${ }^{25}$. La técnica pertenecería al

22 La misma idea la encontramos en J. DELORS et al., La educación, op. cit., 12-13 y 20.

23 ARISTÓTELES, Metafísica, Gredos, Madrid, 2014, 1048b 25-30; Ética a Nicómaco, Gredos, Madrid, 2014, 1094a 1-5.

24 L. POLO, Obras Completas XIX. Persona y libertad, Eunsa, Pamplona, 2017, 181-242.

25 El radical clásico o de la naturaleza humana descubre que hay una realidad que el ser humano recibe, que es su naturaleza, y evidencia que la verdad no es creación humana, pues lo verdadero es estable. También advierte que hay algo inmaterial en el ser humano que supera el tiempo, y 
radical moderno, luego sin ella no se entiende al ser humano y su actuar. Pero al mismo tiempo nos descubre que si la técnica no depende del carácter personal se vuelve contra el hombre.

Para las pretensiones del artículo, la crítica al modelo competencial coincide con la crítica al radical moderno y la propuesta de la educación centrada en la relación depende de la propuesta poliana de entender los tres radicales unidos jerárquicamente por el radical personal. Esto es determinante para nuestra propuesta y permite descubrir que la crítica al modelo competencial no puede confundirse con la crítica a la competencia. La competencia tiene su lugar en la educación, como el radical moderno tiene su lugar en la educación, pero no puede tomarse la competencia como base, como no puede tomarse el radical moderno como base. Eso supone un error de consecuencias negativas, que no puede solucionarse "añadiendo" los otros dos radicales, sino que hay que hacer un cambio para que la base sea el radical personal y desde ahí los otros dos tendrán su lugar. También Delors decía que no era cuestión de sumar 'pilares' sino que el de aprender a vivir juntos era el central.

Decíamos que otro elemento de Polo es la alta valoración de la educación de la familia. Ésta resalta la conexión entre educación y relaciones interpersonales pues, como hemos dicho, la educación descansa en nuestra condición de hijos.

Para nuestro interés conviene destacar la educación de la imaginación porque es garantía de que la maduración ha de ser global. Esto se nota en que la maduración de un aspecto aislado de los demás no es posible ya que por ejemplo, sin imaginación (que nutre a la cognición) no se puede producir y desarrollar proyectos y, por otro lado, la educación de la afectividad acabaría en fantasía.

Por último, señalar que la motivación descansa en la motivación trascendente, es decir, llegar a ser de una forma concreta. Así la motivación es realmente interior. Y también lo será su evaluación, frente a la competencia que ésta es evaluada por algo que ocurre fuera de la persona.

Francisco Altarejos es un filósofo de la educación que comparte con Leonardo Polo las afirmaciones centrales que sirven para estructurar el acto edu-

que al poner en acto sus facultades éstas mejoran. El ser humano es acto en sus actos. El radical moderno o el del sujeto es productivo y aporta la novedad cultural. Pero 'producir' no es 'causar', porque el efecto de la causa no comporta novedad. El radical cristiano o personal, señala que se está llamado a trascenderse, a encontrarse con las demás personas creadas e increadas. 
cativo. Éstas son: La diversidad de crecimientos y la singularidad del crecimiento humano calificado de irrestricto ${ }^{26}$, la forma de entender la libertad y la autodeterminación originaria en todas las elecciones que realiza ${ }^{27}$, la antropología transcendental ${ }^{28}$ y el concepto de hábito ${ }^{29}$. La sintonía conceptual entre ambos autores puede apreciarse en el prólogo que Altarejos hace del ensayo de filosofía de la educación escrito por Polo en "ayudar a crecer" ${ }^{30}$, como en las constantes referencias que Altarejos hace a la obra de Polo en sus escritos. Estos principios enunciados junto con el transcendental descubrimiento de Aristóteles que permite diferenciar el acto de la actividad y que Altarejos usará para calificar la educación de "acto" serán fundamentales para entender su postura sobre la misma.

Altarejos sitúa la educación en el ámbito de la naturaleza humana, puesto que es la misma naturaleza la que reclama que se dé la educación. Nacer no formado e indeterminado es una singularidad del ser humano, no por deficiencia, sino precisamente porque muchas funciones le son posibles. Su naturaleza racional permite el crecimiento irrestricto pues ésta nunca se limita ni $\operatorname{agota}^{31}$.

Una característica fundamental de Altarejos es lo que podríamos llamar una propuesta sistémica. El término sistema es presentado por Polo, en un sistema "al modificarse uno (de sus elementos) se modifican los demás" que, o se considere todo en su conjunto y unidad, o se deformará la realidad. Altarejos indica que por la suma ordenada y con una jerarquía se puede llegar a un todo ordenado, pero nunca a una realidad integrada. En la propuesta de Altarejos se parte de la integración y se crece $^{33}$. Esta consideración es determinante en su propuesta y que puede tomarse como punto de referencia seguro a la hora de las críticas que hace a otras propuestas educativas. Asumir la concepción sistémica nos lleva a nivel educativo a centrarnos en la relación y no en el alumno, sencillamente porque no existe el alumno aislado de la misma forma que no existe la persona aislada. La consideración social no es que

\footnotetext{
F. ALTAREJOS, C. NAVAL, Filosofía de la educación, 17.

Ibidem, 125-128.

Ibidem, 151-178.

Ibidem, 191-200.

L. POLO, Ayudar a crecer, op. cit.

F. ALTAREJOS, C. NAVAL, Filosofía de la educación, 17-23.

L. POLO, ¿Quien es el hombre?, op. cit., 67.

F. ALTAREJOS, C. NAVAL, Filosofía de la educación, 86.
} 
sea importante porque es una realidad que influye. Las relaciones interpersonales son más que una influencia son constituyentes, pues la persona es co-ser.

De esta postura surge una consecuencia clara: no se solucionan las deficiencias de un modelo añadiendo elementos pretendiendo suplir las deficiencias. La crítica que realiza Altarejos recuerda a la crítica al radical moderno en el estudio de los tres radicales de Leonardo Polo ${ }^{34}$. Polo indica que una propuesta filosófica fundada en el radical moderno no puede aprovecharse sin antes no se corrige. Mientras la filosofía clásica puede completarse y desarrollarse, la moderna no hay forma de ello sin la corrección previa. La corrección supone la unión jerárquica de los tres radicales poniendo como culmen el radical personal. Altarejos va a resituar muchos dilemas educativos desde la perspectiva personal, lo cual le permite corregir errores y ofrecer una propuesta mucho más consistente.

Esto nos permite a nosotros afirmar que una educación que considere seriamente el carácter personal del ser humano necesita entender la educación como centrada en las interrelaciones personales.

Son más los puntos en los que pueden descubrimos encuentros entre el pensamiento de Altarejos y Polo, aunque éstos no se mencionen explícitamente. Los mencionamos porque pensamos que Altarejos ha desarrollado a nivel educativo lo que Polo esbozó en su ensayo 'ayudar a crecer'. Por ejemplo, Altarejos insiste en que la retórica es muy importante para la educación, pues la retórica resalta que la educación tiene que persuadir ${ }^{35} \mathrm{y}$, en ese sentido podríamos decir que la educación es atractiva. Esto requiere que el actuar humano surge desde la interioridad de la persona y no es un mero comportamiento reactivo. Que la persona tenga interioridad e intimidad tiene sus implicaciones al considerar las motivaciones en educación, pues junto a una motivación que se entiende como cubrir necesidades, aparece otra motivación que descansa en la libertad. Así aparece la distinción entre fines naturales y fines libres que no pueden entenderse como necesidad. Ambos fines están "mutuamente imbuidos" ${ }^{{ }^{36}} \mathrm{y}$ son los fines libres los que dan sentido a los fines naturales $^{37}$. Esto es así porque el ejercicio de la libertad es la autodeterminación.

\footnotetext{
L. POLO, Persona y libertad, op. cit., 181-242.

F. ALTAREJOS, C. NAVAL, Filosofía de la educación, 40; F. ALTAREJOS, Dimensión ética de la educación, Eunsa, Pamplona, 1999, 89.

36 F. ALTAREJOS, C. NAVAL, Filosofía de la educación, 80.

37 F. ALTAREJOS, C. NAVAL, Filosofía de la educación, 77-80.
} 
La autodeterminación no debe entenderse como alcanzar una vida en concreto. De ser así, se estaría confundiendo con cumplir un plan, con fines concretos. Si se educa para la vida y la vida no puede identificarse con los fines concretos, tampoco la educación puede tener un fin concreto ${ }^{38}$. Dicho en palabras de Altarejos cuando resumió parte del mensaje de Polo "el hombre es un ser de proyectos porque él es un proyecto" ${ }^{\prime 3}$. El carácter de persona del ser humano (su ser irrepetible e irreductible) hace que el ser humano no se agote en todo lo que es, puede más y de ahí que se hable de la educación como formación ${ }^{40}$ $\mathrm{y}$ todo fin concreto se le quede corto.

Lo cual, a su vez y en terminología de Polo, requiere entender que la persona es intimidad y desde ella se expresa. Porque estrictamente hablando, con la educación no se forma a nadie, sino que se ayuda para que el alumno se forme a si mismo ${ }^{41}$.

Otro punto de apoyo decisivo para su propuesta educativa será aplicar el descubrimiento del acto según Aristóteles a la educación. Frente a una educación conceptualizada como actividad y centrada en el resultado, la educación como acto implica que el acto educativo tenga valor por lo que está aconteciendo en ese mismo momento y no por lo que sucederá después ${ }^{42}$. En la propuesta de Altarejos, acto no se opone a actividad, sino que, en el vivir propiamente humano, el acto y la actividad coexisten, pero es el acto el que marca la calidad humana de la actividad. De Aristóteles, Altarejos también toma la referencia al felicidad como el fin final que debe de estar presente en todo fin (parcial). El fin final es la referencia a la felicidad ya que al ser el fin último del hombre, debe serlo igualmente de la educación ${ }^{43}$. La importancia de esto reside en que de la suma de fines parciales nunca se llega al fin final. Si el fin final no está presente en toda actividad, entonces se cae en la disgregación de los fines y cada uno de ellos acaba por perder su sentido. Desde los fines no se llega al fin final, pero si al revés ${ }^{44}$. Pues por la suma no se llega a la unidad ${ }^{45} \mathrm{y}$ los

\footnotetext{
38 F. ALTAREJOS, C. NAVAL, Filosofía de la educación, 104.

39 F. ALTAREJOS, "Leonardo Polo: pensar la educación”, en Ayudar a Crecer, Eunsa, Pamplona, 2006, 2.

40 F. ALTAREJOS, C. NAVAL, Filosofía de la educación, 153-157.

${ }^{41}$ Ibidem, 179.

42 F. ALTAREJOS, Dimensión ética de la educación, 79-94; F. ALTAREJOS, C. NAVAL, Filosofía de la educación, 33-35.

43 F. ALTAREJOS, Educación y felicidad, Eunsa, Pamplona, 1983.

44 F. ALTAREJOS, C. NAVAL, Filosofía de la educación, 89.

45 F. ALTAREJOS, C. NAVAL, Filosofía de la educación, 86-90.
} 
diversos fines lo son, porque participan del fin final ${ }^{46}$. Esto guarda relación con la propuesta teleológica de la acción humana que ya formuló Aristóteles y que Altarejos asume ${ }^{47}$.

El tratamiento que da Altarejos al fin final y su relación a los diversos fines ed fundamental para nuestra propuesta de la educación centrada en la relación interpersonal. Si Altarejos afirma que el fin final debe hacerse presente en cada fin, nosotros nos preguntamos ¿cómo puede hacerse eso? La respuesta sería por la relación misma entre el profesor y el alumno. Cuando se le dice al alumno 'estudia que esto será bueno para tu futuro' aparece el problema no sólo de saber esperar lo cual pueda aprenderse, sino que el verdadero problema es que el fin final no se hace presente. Estudiar algo no se justifica por cuestiones prácticas de que algo bueno puede ocurrir en el futuro. Si no es bueno ahora, no hay porque pensar que lo será luego. Si lo que se estudia ahora, por la forma de estudiar, no sirve para mejorar las relaciones sociales (profesor-alumno-alumnos-familia) ¿por qué ha de servir luego? Si el fin final no se hace presente ahora, no hay garantía de que se dé luego. No se estudia matemáticas para hacer grande las matemáticas, sino porque las matemáticas sirven para mejorar las relaciones sociales. Y si ahora no sirven para mejorar las relaciones sociales entre profesor-alumnoalumnos-familia ¿por qué ha de ocurrir luego? No es razonable pensarlo. Así pues detrás del inconformismo de muchos alumnos puede encerrarse (junto con otras muchas cosas) una exigencia de una educación de calidad de verdad.

Un elemento que es crucial en Altarejos y para el propósito de nuestra propuesta de la educación centrada en la relación interpersonal es el carácter social de la educación con la que supera con mucho lo indicado por Vygostky o Paulo Freire. Lo supera porque el carácter social no pertenece simplemente a la naturaleza, sino que depende del acto de ser personal. Sin tener detrás el transcendental personal de la co-exitencia de Leonardo Polo resultará imposible entender la transcendencia del presente punto y del artículo. La persona sólo es persona por su relación con otras personas ${ }^{48}$. Co-existencia que romperá la autoafirmación de la cerrazón de la subjetividad pues reclama la autotranscendencia para poder llegar a ser lo que se está llamado a ser en el

\footnotetext{
F. ALTAREJOS, C. NAVAL, Filosofía de la educación, 90.

F. ALTAREJOS, C. NAVAL, Filosofía de la educación, 32.

48 F. ALTAREJOS, C. NAVAL, Filosofía de la educación, 161-163.
} 
encuentro solidario con el otro ${ }^{49}$. Para Altarejos, la comunicación no es meramente compartir información, sino que la comunicación es una coparticipación de las dos personas, donde el propio "yo" es compartido y requiere de ser acogido y reconocido ${ }^{50}$. La comunicación objetiva (que tiene formato de actividad según terminología aristotélica) requiere de la comunicación subjetiva, y además la objetiva se subordina a la subjetiva (como la actividad al acto $)^{51}$. Donde se descubren todas las implicaciones del carácter co-existente aplicado a la educación es cuando Altarejos expone cual es el lugar e importancia de la familia ${ }^{52}$. No es que la familia sea importante por la repercusión educativa que de hecho tiene en las personas o por los beneficios que esto pudiera conllevar, sino que la familia es el modelo de lo que significa educar. Educar es un engendrar segundo. Se ha roto toda posibilidad de individualismo y el educador queda inmerso en el proceso educacional, porque también él/ella tiene que aprender. Los padres aprenden la humanidad de sus propios hijos. Frente a una educación individual concebida como adaptación y espontaneidad, la familia es modelo de educación relacional. La escuela debería pues asemejarse a la familia, ya que citando a J. Rassam, Altarejos repetidamente señala que "se educa por lo que se es, más por lo que se dice. Se enseña también lo que se es más que lo que se sabe" y se valora altamente el crecimiento en la relación interpersonal como vía educativa para descubrir "al hombre

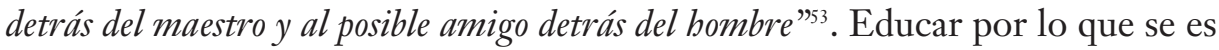
lleva a una unidad de razón y voluntad, educar por lo que se dice lleva a la disgregación de la realidad del ser humano y a una visión tecnificada de la educación.

Con esta breve presentación de su pensamiento mezclada con nuestras reflexiones, su definición de educación puede comprenderse mejor. Educar es "la acción recíproca de ayuda al perfeccionamiento bumano, ordenado intencionalmente a la razón, y dirigido desde ella, en cuanto que promueve la formación de bábitos éticamente buenos ${ }^{\prime 4}$.

49 F. ALTAREJOS, "La co-existencia, fundamento antropológico de la solidaridad (K. Wojtyla, R. Spaemann, L. Polo)", en Studia Poliana, 8 (2006), 119-50.

50 F. ALTAREJOS, C. NAVAL, Filosofía de la educación, 47-48; F. ALTAREJOS, Dimensión ética de la educación, 27-59.

51 F. ALTAREJOS, C. NAVAL, Filosofía de la educación, 51-54.

52 F. ALTAREJOS, Dimensión ética de la educación, 165-178.

53 J. RASSAM, "Le professeur et les élèves", en Reveu Thomiste, 76 (1979), 64.

54 F. ALTAREJOS, C. NAVAL, Filosofía de la educación, 31. 
Desde estos principios se entienden muchas de las posturas de Altarejos presentando una visión completa y unificada, no por agregaciones sino por una visión holística ${ }^{55}$. Por ejemplo Altarejos postula que no pueda educarse a la persona entera educando sus "partes", sino educándola a toda ella entera en su unidad ${ }^{56}$. Tampoco es posible asumir el modelo regulador de la educación emocional sino que hace falta un modelo integrador ${ }^{57}$. La instrucción no tiene sentido fuera de la formación, ni el aprendizaje sin la formación, ni la inteligencia sin la voluntad ${ }^{58}$. Ni el saber teórico, sin el práctico, pues su distinción es de naturaleza intelectual sin separación real y en educación no dejan de ser diversas perspectivas ${ }^{59}$.

Este recorrido sirve para entender dos visiones de la educación totalmente contrapuestas: una educación centrada en la eficacia frente a una centrada en la libertad ${ }^{60}$; una centrada en la información frente a una centrada en la comunicación interpersonal ${ }^{61}$; una centrada en el dato (competencias como resultado) frente a otra centrada en el símbolo (perfeccionamiento subjetivo) ${ }^{62}$; una que secuencia en momentos independientes la voluntad y la razón frente a otra que operan unificadamente, o una voluntad dirigida desde fuera (en sus diversos grados: premio, castigo, poder, afectos, deseo) frente a una voluntad operante desde el interior (el encuentro interpersonal buscando la unidad con el otro respetando su alteridad $)^{63}$; una educación como mera actividad u otra como acto-actividad ${ }^{64}$.

Tras la presentación comentada de Polo y Altarejos veamos cinco autores más que sustentan principios en parte similares y sirven, por tanto, de refuerzo de las ideas precedentes. Todos ellos pueden leerse en clave de confirmación de la propuesta de Polo y Altarejos porque obligan a una visión holística y sistémica de la educación por lo que no es posible centrarse en la

55 Holístico se distingue de comprensivo en la referencia a la unidad. Un tratamiento comprensivo es que trata de todo, pero no tiene por qué tratarlo desde la unidad. La propuesta holística lo hace desde la unidad.

56 F. ALTAREJOS, C. NAVAL, Filosofía de la educación, 22.

57 F. ALTAREJOS, "Autorregulación e integración: dos propuestas en la educación de la afectividad (D. Goleman y Tomás de Aquino)", en Estudios sobre Educación, 7 (2004), 45-66.

58 F. ALTAREJOS, C. NAVAL, Filosofía de la educación, 37-43.

59 F. ALTAREJOS, C. NAVAL, Filosofía de la educación, 70.

60 F. ALTAREJOS, Dimensión ética de la educación, op. cit., 17-26.

61 Ibidem, 27-42.

62 Ibidem, 43-58.

63 Ibidem, 59-78.

64 Ibidem, 79-94. 
mera persona del alumno, pues la persona lo es en un plexo de relaciones como realidad constituyente. Todos los autores siguientes sirven para negar una visión técnica de la educación como si ésta se jugara en un aspecto de la persona aislado de los demás. Así pues veamos como los diversos autores consideran que la educación debe atender a la complejidad unitaria y dinámica de la persona y por tanto, rechazan una visión técnica o sectorial.

En la propuesta de Víctor García Hoz también queda muy claro que la educación no es algo accesorio sino que pertenece al mismo ser perfectible del ser humano "La causa material de la educación es la perfectibilidad bumana" pues cuando nace "está sin hacer" ca el desarrollo humano, sino en aquello que le es específico y le transforma para ser plenamente lo que se es ${ }^{67}$. García Hoz realiza su exposición siguiendo la tetracausalidad. Lo interesante de esta apuesta ${ }^{68}$ es que al ser obligatorio entender la tetracausalidad en intrínseca relación y unidad de las cuatro causas, es imposible caer en la fragmentación del acto educativo. Así se evita caer en una visión técnica de la educación pues al tener que considerar la causa final en el acto educativo indica que "el fin de la educación ha de estar ligado al fin del hombre" ${ }^{\text {"69 }}$ a su propia perfección como hombre. Rechaza una educación utilitarista para centrarse en "las virtudes como fin de la educación"

Octavi Fullat mantiene tesis similares a Polo y Altarejos al indicar que " $\mathrm{el}$ hombre es una realidad inacabada e inexorablemente abierta" ", lo cual hace que el hombre sea su principal proyecto "ser humano es inesquivablemente producir$s e{ }^{m / 2}$. Esto requiere entender la educación en clave de crecimiento y en una dirección a un "para qué"ris que no es otra cosa que llegar a ser plenamente ya que "educar es engendrar a lo bumano"

$\mathrm{Al}$ igual a Polo, Fullat sostiene que la mera naturaleza no podemos comprender todo lo que el ser humano es y por eso añade la referencia a la liber-

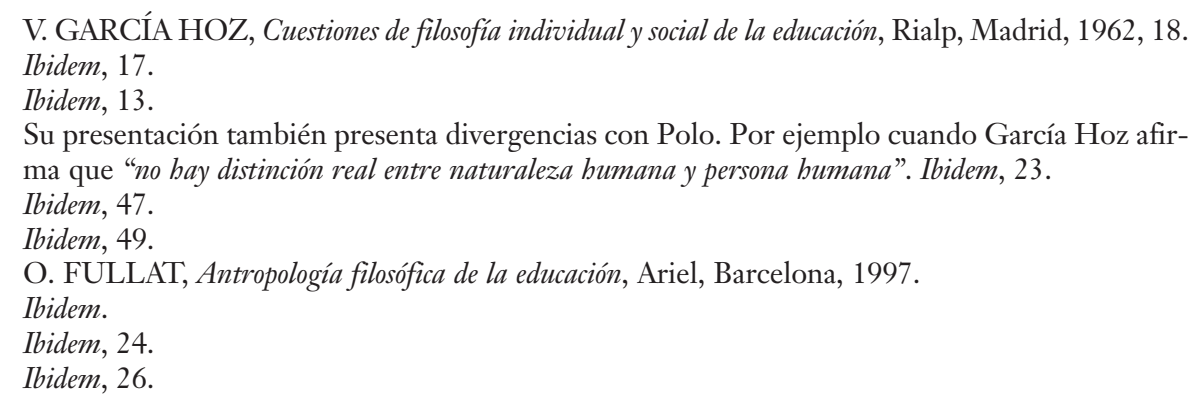


tad "parece sensato sostener que el ser humano no puede reducirse a naturaleza disfrutando también del espacio de la libertad" ${ }^{\prime 5}$ y también califica al hombre de "sistema abierto" $"$.

Fullat valora la técnica, pues nuestra forma de estar en el mundo es cambiándolo ${ }^{77}$, pero rechaza cualquier visión neutra de la técnica, pues en el acto humano la persona entera se hace presente. De ahí la urgencia de globalidad y para entender la educación reclama contar con la naturaleza, la cultura y el espíritu humano. Afirmar que el hombre es espíritu es afirmar que es "exceso y desmesura" ${ }^{\text {}}$. Esto hace que cualquier conocimiento, por muy excelso que sea será siempre limitado, pues el hombre es siempre más.

Uno de los grandes referentes de la filosofía de la educación es Richard Stanley Peters quien diferencia claramente entre el "bombre entrenado" y el "bombre educado" al plantear que el propósito de la educación permite a los hombres y mujeres ser mejores, y esto no lo consigue un mero entrenamiento de habilidades. La educación apunta a una forma de vida, una visión del mundo y de la vida con sentido por sí misma y que no puede ser conceptualizada de forma utilitaria ${ }^{79}$.

Aunque Peters no utiliza el término de 'acto' aristotélico sí que se refiere a la educación de una forma congruente a esta visión. Pues, la educación tampoco puede reducirse a un tipo de actividad concreta ${ }^{80}$ sino que la actividad se vuelve educativa si considera lo valioso en sí y su desarrollo implique lo moral, pues el éxito es la virtud ${ }^{81}$.

La referencia a lo valioso en sí, pensamos que remite o reclama el término 'acto' de Aristóteles pues tiene el fin en sí mismo, no fuera de. Esto es fundamental para nuestra propuesta de la educación centrada en la relación, pues el valor del acto educativo no debería estar por algo que ocurriera fuera de la clase, sino que la misma clase tendría que ser valiosa en sí. Y en el ser humano algo es valioso porque sirve para la perfección de las relaciones interpersonales. Esto rechaza una visión técnica de la educación y la posibilidad de que el profesor permanezca a cierta distancia del proceso. El pro-

\footnotetext{
Ibidem, 34.

Ibidem, 55.

Ibidem, 75.

Ibidem, 147.

S. PETERS, Ethics and education, op. cit.; "What is an educational process", op. cit.

S. PETERS, Ethics and education, op. cit., 24-25.

Ibidem, 26.
} 
fesor debe de estar inmerso en el proceso educativo como alguien que también crece, pues si el crecimiento de la persona es el crecimiento de sus relaciones interpersonales entonces, el profesor también crece en ese acto educativo.

La cognición (que no es un mero saber, sino conocer causas, principios y capacidad crítica) se supedita también al crecimiento pues debe ser un conocimiento que transforme a la persona considerada en su globalidad ${ }^{82}$. Luego la cognición no es un acto técnico.

También desarrolla el principio de experiencia pero evita su aislamiento. El camino de la experiencia inicia un desarrollo en el que lo emocional, la conciencia, las creencias, lo significativo se van formando y diferenciando "pari passu $^{\text {"s3. }}$. Es decir, al mismo ritmo y nivel lo cual reclama que todas ellas se pongan en juego.

No hay progreso personal al margen del crecimiento social ya que liga la autorrealización con lo social para promover la integración social amando y siendo amado generando fraternidad por lo que centrarse en la competencia es un error ${ }^{84}$. Más aún, sostiene que una motivación centrada en el dominio, la competencia y la manipulación puede degenerar en compulsión y obsesión si no están intrínsecamente ligadas a algo valioso que les dé sentido ${ }^{85}$. Lo contrario a la propuesta de Peters es el utilitarismo del cual surge la visión de la diversidad de tareas independientes en el acto educativo ${ }^{86}$ ya que cada intervención tiene un fin útil centrado en algo exterior a la persona.

Por último, Peters tiene una visión bastante completa de lo humano y el acto educativo. Ya que el motivo que tiene para rechazar ciertas propuestas educativas es precisamente que se tratan de consideraciones reductoras de la complejidad de lo humano ${ }^{87}$. El referente ético lo despliega en una serie de principios éticos que sirven para determinar el currículo. Éstos son: igualdad, lo valioso, el interés, la libertad, el respeto y la fraternidad ${ }^{88}$.

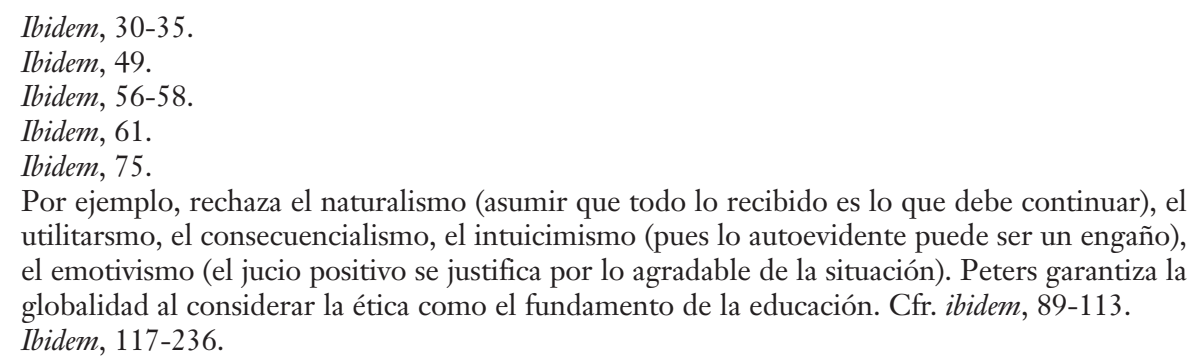
utilitarsmo, el consecuencialismo, el intuicimismo (pues lo autoevidente puede ser un engaño), el emotivismo (el jucio positivo se justifica por lo agradable de la situación). Peters garantiza la globalidad al considerar la ética como el fundamento de la educación. Cfr. ibidem, 89-113. Ibidem, 117-236. 
Esta visión completa de la educación Peters la entiende como normativa incluso para los años universitarios, incluso en carreras marcadamente técnicas ${ }^{89}$.

La propuesta de Whitehead refuerza la línea que describimos porque presenta una comprensión global de la educación e impide reduccionismos técnicos. La educación y el crecimiento del organismo no son realidades independientes entre sí: existir, vivir, obrar, etcétera es lo mismo. Frente a una visión intelectualizada de la razón, Whitehead une la razón a la vida, pues la función de la razón no es otra que promover el arte de vivir $^{90}$. La clave de la vida es maximizar la satisfacción de vivir ${ }^{91}$. Por lo que la educación no es nada externo a la persona, ni se mide por algo externo a ella. Su apuesta es por la vida buena que es la vida mejor ${ }^{92}$. Se educa para vivir bien y mejor, no para sacar utilidad a la vida y cualquier desarrollo cognitivo se pone al servicio del arte de vivir ${ }^{93}$. Por ello cualquier división entre vida y escuela es artificial, pues el desarrollo intelectual está al servicio del desarrollo ${ }^{94}$. Rechaza interpretaciones cognitivistas o utilitaristas (hacer algo por el beneficio recibido) ya que la utilidad está en al arte de vivir.

También se ve la globalidad de su propuesta cuando indica que "la esencia de la educación es ser religiosa ${ }^{\$ 55}$ hay que calificar que Whitehead no se identifica con ninguna religión ni Dios. La referencia a lo religioso debe entenderse como la perfecta y posible realización del proceso que genera un atractivo. Esto descubre que la educación debe apuntar a lo más alto de las posibilidades humanas y por otro lado, que la educación tiene que tener un carácter propositivo, pues según su propuesta, Dios no se impone sino que atrae por su atractivo.

Si unimos las dos afirmaciones clave de Whitehead: la maximización de la experiencia y la visión orgánica del ser humano ${ }^{96}$ llegamos necesariamente a incluir al profesor como alguien que experimenta un cambio en el proceso educativo. La visión del organismo hace que sea imposible entender al alumno fuera de su plexo de relaciones en las que obviamente entra el profesor con

\footnotetext{
Ibidem, 68-72.

A. N. WHITEHEAD, The Function of the Reason, Princeton University Press, Princeton, 1929, 2. Ibidem, 23.

Ibidem, 30.

A. N. WHITEHEAD, Los fines de la educación y otros ensayos, Paidós, Buenos Aires, 1957, 20.

Ibidem, 15.

Ibidem, 34 .

A. N. WHITEHEAD, Process and Reality. An Essay of Cosmology, The Free Press, New York, 1978.
} 
el que tanto tiempo pasa. Esa experiencia de relación es lo que constituye la actualidad del alumno y el crecimiento del alumno consiste en maximizar la experiencia constituyente de sus relaciones interpersonales. Lo mismo podría repetirse del profesor. Pensamos que esto es otro refuerzo clave a nuestra propuesta de la educación centrada en la relación interpersonal.

La propuesta de Lawrence Kohlberg interesa por dos motivos. Porque entiende que el desarrollo de la persona es el objetivo de la educación ${ }^{97}$ y porque la visión de un profesor-entrenador es imposible en su propuesta, pues la clave está en la vida compartida entre el profesor y el alumno. Aunque la propuesta inicial de Kohlberg es más cognitivista (basadas en el razonamiento teórico del estudio de casos) la abandonó por considera que ésta es limitada ${ }^{98}$. Su nueva propuesta es "la comunidad justa" (just community)". La comunidad justa convierte la comunidad escolar en comunidad de vida pues se centra no en la adquisición de conocimientos, sino en compartir la misma vida de las personas. La distancia entre la escuela y la vida se anula, pues en la escuela se vive la vida que se quiere promover.

Finalmente hacemos un apunte a Malcom Knowles que es un referente muy importante para la educación de adultos. Podría pensarse que la educación personalista que se está sosteniendo tendría que restringirse a la primaria y secundaria, pero que una vez que la persona ya está formada se podría pasar a una educación en clave más técnica. En cambio encontramos que la propuesta que hace Knowles (apoyándose sobre todo en psicólogos humanistas como Rogers y Maslow) mantiene las claves personalistas indicadas hasta el momento. Su propuesta es la 'adragogía' en la cual el aprendizaje del alumno se entiende como un proceso autodirigido que supera las meras habilidades y conocimiento para centrarse en el desarrollo/crecimiento personal y la enseñanza del profesor la entiende como un ayudador o facilitador del crecimiento sobre todo gracias al estilo de relación que establece con el alumno y él también crece. Hace esta propuesta en contraposición a una visión, que el observa como dominante en su entono, centrada en adquirir habilidades y conocimiento siguiendo las instrucciones de otro y una enseñanza donde el docente es un instructor que permanece al margen del proceso, pues el que debe

\footnotetext{
97 L. KOHLBERG, R. MAYER, "Development as the aim of education”, en Harvard Education Review, 4.4 (1972), 449-96.

98 C. F. POWER, A. HIGGINS, L. KOHLBERG, Lawrence Koblberg's approach to moral education, Columbia University Press, New York, 1989, 33-34.

99 Ibidem.
} 
cambiar es el alumno ${ }^{100}$. Su investigación progresa hasta concluir que la andragogía no debería de ser la propuesta para el adulto, sino que se trata de una forma de considerar los procesos de enseñanza/aprendizaje que debería de ser válido con independencia de la edad ${ }^{101}$.

\section{LA PROPUESTA DE LA EDUCACIÓN CENTRADA EN LA RELACIÓN}

Vemos que se está dibujando otro modelo educativo alternativo al modelo basado en las competencias que, siguiendo la propuesta de Delors de jerarquizar los pilares desde el aprender a vivir juntos, proponemos llamar educación centrada en la relación interpersonal. Las notas que identifican la propuesta sería la siguiente:

1. La necesidad de introducir la visión sistémica al considerar lo humano y su educación.

2. Ligar la educación a la naturaleza humana y, por tanto, no centrarse en una edad concreta, sino que toda la vida ha de vivirse en clave de educación.

3. Acentuar que el acto humano mana desde el interior de éste y que ninguna actividad puede entenderse al margen del acto.

4. Si la educación es ayudar a ser persona, la educación debe ser estructurada desde el carácter personal, lo que a su vez implica, que la educación es permitir que la co-existencia, el conocer, la libertad y el amor donal encuentren su desarrollo.

Con la formulación de una educación centrada en la relación interpersonal se quiere recoger estas cuatro notas y si hace la apuesta por acentuar la relación interpersonal es para imposibilitar cualquier reducción utilitarista o centrada en algo exterior a la persona. Tampoco esta propuesta escapa de la posibilidad de ser mal interpretada, como pensamos que lo fue la propuesta de centrada en el alumno cuando se concretizó en el modelo competencial. En ese sentido la propuesta del modelo de centrado en la relación no es tanto algo en oposición al modelo centrado en el alumno sino una clarificación para evitar caer en el modelo competencial. Además también el modelo de educación

${ }^{100}$ M. KNOWLES, "What is andragogy?", en The Modern Practice of Adult Education. From Pedagogy to Andragogy. Revised and Update, Cambridge Adult Education, New York, 1980, 40-61.

101 M. KNOWLES, The Adult Learner: A Neglected Species, Gulf Publishing Company, Houston, 1973. 
centrada en la relación, como todo modelo, puede degenerar. La degeneración de la propuesta centrada en la relación interpersonal sería exagerar la experiencia emocional del encuentro interpersonal. Caeríamos así en una afectivación de la educación. Esto supondría la misma destrucción de la propuesta, pues llevaría a centrarse en uno mismo.

Con el recorrido hecho es evidente que el fin final debe de estar presente en cada acto educativo, también se descubre que el acto marca la calidad de toda actividad, como que el fin final da sentido a todos los fines. Si esto lo unimos al hecho de que la familia es el modelo de educación, es entonces evidente, que el profesor, en cuanto persona, no puede quedar al margen del acto educativo. Una visión del profesor como suporte y conductor, según el modelo de scaffolding de Vygostky sería pobre. El mismo profesor está llamado a crecer. Incluso podríamos llegar a decir, si el profesor no crece, tampoco crecerá el alumno, pues el crecimiento de la persona es el crecimiento de sus relaciones interpersonales porque decir persona es decir relación desde la intimidad. Sólo el humano tiene intimidad y llamamos al humano persona porque establece relaciones de intimidad a intimidad. Tal es la visión de Leonardo Polo sobre la persona. La calidad de la relación entre el educador (bien padre, bien profesor, bien cualquier persona) y el educando marcará la calidad de la educación. Además la educación no se circunscribe a un periodo de la vida, para suplir una deficiencia, sino para expresar un modo de ser y de relacionarse. Todo encuentro humano debería de estructurarse bajo la clave educativa de ayudar a crecer en la interrelación personal. La frontera entre escuela y vida queda disuelta.

Estas notas al concretarse nos van dibujando una visión de la educación que se contrapone a una educación centrada en las competencias. La competencia en terminología de Polo la hemos situado en el radical moderno. Si el radical moderno se aísla es cuando surge el modelo competencial y con él los problemas asociados. Si el radical moderno se pone dentro del sistema de los tres radicales jerarquizados por el radical personal, entonces la competencia tiene su lugar y en lejos de entorpecer favorece el crecimiento personal.

El objetivo de la educación se relaciona con la maduración personal en la vida real afrontando los retos concretos de cada edad y respondiendo a una llamada interior. El medio por el que se realiza la educación coincide con el fin pues es el encuentro interpersonal y su mejora. Así actuando sobre las propias relaciones interpersonales en situaciones de realidad (los problemas que plantea el vivir de cada día) es como la persona crece. Por ejemplo, el trabajo en grupos estaría centrado en qué hacer para mejorar las relaciones interperso- 
nales de los miembros del grupo y los que están fuera de él. Las diversas asignaturas son diversas plataformas para el crecimiento interpersonal. Los problemas propios del sistema competencial como la transferencia de lo aprendido a la vida real dejarían de ser un problema, pues desaparece la frontera entre escuela y vida. Además, como el actuar nace de dentro de la persona, el principio de actuación no cambia en función de dónde se esté. La motivación se centra en el desarrollo personal que implica una forma de ser y vivir en el mundo y en un desarrollo personal, que no es distinto del desarrollo de las relaciones interpersonales. El valor de la persona es incondicional y el educador es más que lo tradicionalmente visto como su rol o función, pues incluye la experiencia de su propio crecimiento personal. El profesor también cambia y está llamado a crecer.

Este debate entre el modelo basado en competencias o el modelo centrado en el crecimiento de las relaciones interpersonales no es nuevo. Hay motivos suficientes para pensar que el debate está desde el mismo nacimiento de la escuela. En tiempos de Platón y Sócrates estaba la disputa de si convenía enseñar o no dialéctica a los jóvenes. A Sócrates, recordemos, lo trataban de corruptor de la juventud. El problema que Platón denunciaba en su libro "la República"102 estaba en que la educación se había tecnificado pues la dialéctica se había visto como una herramienta que estaba siendo usada para la confrontación. Las creencias y la propia vida habían quedado al margen. Los jóvenes que aprendían la dialéctica, siguiendo el camino de sus maestros, acaban de ordinario por no creer en lo que creían y surge la malicia. Educar desligado de la vida, de la relación y de las creencias lleva a este panorama. En cambio Sócrates, en contraposición a los otros dialécticos profesionales, se esforzaba en ligar escuela y vida ${ }^{103}$.

Pensamos que esa misma confrontación de modelos de educación persiste entre el modelo basado en competencias frente a una educación de corte personalista que es lo que pretendemos ofrecer bajo el título de una educación centrada en ayudar a crecer las interrelaciones personales. Propuesta que pensamos que es una conclusión lógica del estudio del documento de la UNESCO y de la propuesta de Polo-Altarejos y apoyada en otros filósofos de la educación.

102 PLATÓN, República, Instituto de Estudios Políticos, Madrid, 1969, VII-VIII.

${ }^{103}$ Puede encontrarse una explicación más detallada de estas ideas en: M. LIPMAN, A. M. SHARP, F. S. OSCAYAN, La filosofía en el aula, Ediciones de la Torre, Madrid, 2002, 31-36. También en F. GARCÍA MORIYÓN, Pregunto, dialogo, aprendo. Cómo hacer filosofía en el aula, Ediciones de la Torre, Madrid, 2006, 34. 
RESEÑAS Y NOTICIAS REVIEWS AND NEWS 